\title{
Causal reasoning and symbolic relationships in Medieval Illuminations
}

\author{
Djibril Diarra $^{1}$, Martine Clouzot $^{2}$, Christophe Nicolle ${ }^{1}$ \\ ${ }^{1}$ Laboratoire Electronique Informatique et Image (LE2I), Burgundy University, France \\ ${ }^{2}$ Laboratoire Archéologie Terre et Histoire (ArTeHis), Burgundy University, France \\ Corresponding author: Djibril Diarra, dl4djibril@gmail.com
}

\begin{abstract}
This work applies knowledge engineering's techniques to medieval illuminations. Inside it, an illumination is considered as a knowledge graph which was used by some elites in the Middle Ages to represent themselves as a social group and exhibit the events in their lives, and their cultural values. That graph is based on combinations of symbolic elements linked each to others with semantic relations. Those combinations were used to encode visual metaphors and influential messages whose interpretations are sometimes tricky for not experts. Our work aims to describe the meaning of those elements through logical modelling using ontologies. To achieve that, we construct logical reasoning rules and simulate them using artificial intelligence mechanisms. The goal is to facilitate the interpretation of illuminations and provide, in a future evolution of current social media, logical formalisation of new encoding and information transmission services.
\end{abstract}

\section{Keywords}

Symbolic relation; Medieval illumination; Semantic relation; Ontology; Social network

\section{INTRODUCTION}

Knowledge engineering aims to formalise human knowledge so that it can be manipulable by computerised systems. In this paper, its techniques are used to identify, characterise, and to formalise knowledge in the medieval illuminations. Those illuminations are images which were designed for and used by elites in the Middle Ages to represent themselves as a social group and to exhibit the events in their lives and their cultural values. The particular ones took into account in our work are the medieval illuminations of the court of Burgundy's Duke (cf 2.1), Philippe the Good ${ }^{1}$. They constitute a communication system based on symbolic elements (items) linked with semantic and influential relations whose meanings depend on their design's context which is not unique.

This dependence, changing design's context, and the abundance of implicit messages strewed in the medieval illuminations make their understanding difficult and then requires experts' help. Another problem we also faced is the strong correlation between those illuminations' components. Some of those correlations lead to new knowledge which can be inferred from the ones explicitly identified.

\footnotetext{
${ }^{1}$ Philippe the Good (1419-1467) was the most famous of Burgundy Dukes and one of the most powerful European princes at the Hundred Years War's time (1337-1453).
} 


\subsection{Research Objectives and Contributions}

To tackle those problems, we propose to specify and formalise the medieval illuminations' components (symbolic elements, semantic relations, and their meanings) in a knowledge graph which is close to the one which represents current online social networks. Moreover, we are developing a framework based on artificial intelligence mechanisms in order to highlight their implicit meanings and aid to understand them.

Beyond a simple taxonomic modelling, we give more expressivity to the built ontology thanks to the combination of illuminations' components in some reasoning rules which allows our computerised system to reason on the ontology's elements in order to discover new implicit knowledge. Through that system, the experts can express their knowledge about those illuminations and then help in their well understanding by others.

Regard to those objectives, the contributions of our work are double:

- improvement of online social networks: the knowledge graph of our illuminations shows that they have similarities with the current online social networks. Then, the structural comparison between the two graphs could help to improve the current social media's one (e.g. the structural representation of users in order to improve privacy or make a user's social influence evaluation more qualitative);

- digitalisation of cultural heritage's data: the application of knowledge engineering's techniques on the data contained in the illuminations, through the data representation, their storage, integration into other data, and their reuse by computerised system and the aid of artificial intelligence mechanisms in the highlighting of the implicit meaning of illuminations' components could be very helpful for the performances of professionals in the cultural heritage.

\subsection{Organisation of the document}

The rest of this paper is organised as follow. Section II briefly presents our vision on social media and shows the correlation between medieval illuminations and social media, through a detail of their historical context and principle of design. Section III presents some backgrounds of our study. In section IV, an ontological model of illuminations, some examples of formalised semantic relations and textual metaphors are shown as well as an interface of our framework for illuminations' annotation. And section V concludes and gives some future works.

\section{SOCIAL NETWORK AND MEDIEVAL ILLUMINATIONS}

Social networks are the subject of many academic and industrial research projects which deal with different aspects of them: social relations analysis [Wasserman and Faust, 1994, Raad, 2011], sentiment analysis on social network [Martínez-Cámara et al., 2014], implicit communities discovery in social networks [Leprovost et al., 2012], profiles extraction in social network [Ramiandrisoa and Mothe, 2017], information dissemination on social networks [Bakshy et al., 2012], etc. All those works require a large and diversified set of information in different contexts where a social network is considered as a set of relationships which involve regular social interactions between social entities (individuals, organisations, companies, regions, countries, etc) [Wasserman and Faust, 1994]. Those relationships are based on acquaintance, collaboration, collegiality, friendship, etc. They can be direct relationships (an entity $A$ has a direct relationship with an entity $B, A \rightarrow B)$ or indirect $(A \rightarrow B \rightarrow \ldots \rightarrow X$, so $A \rightarrow X)$; symmetric relationships $(A \rightarrow B$ implies $B \rightarrow A$ ) or asymmetric relationships $(A \rightarrow B$ does not involve $B \rightarrow A$ ). Regardless of the type, every social network is maintained by the sharing 
of resources which can be material (e.g. money, livestock, food, equipment, weapons, etc.) or not (e.g. information, strategies, decisions, mood, activities, etc.). The more the reactions of members on the shared resources are high in the network, the more important it becomes. This sharing of resources is one of the fundamental characteristics of every social network either it is represented by a real-life community or by a virtual community: website on the internet such as Facebook ${ }^{2}$, LinkedIn $^{3}, V_{i a d e o}{ }^{4}$, Twitter $^{5}$. In the last case, we call the representation of the social network by social media.

To sum up, a social media is a set of representations (avatars) of individuals or moral people who share texts, images, documents, videos or other media in online computerised platforms within which they are encouraged to interact each with others publicly or in private using the disseminated information. Most of the current social media are structured around the selfvaluation of users in a virtual world they consider as theirs.

\subsection{Illumination: definition and principles of design}

This representation of "me" and "my" environment is known in human history. The rock paintings illustrated a vision which was published through horizontal support, writing or drawing (opposite to oral dissemination, called vertical). In the Middle Ages, to communicate with the elites, the illuminations were developed in books. An illumination is a fixed painting, made with gold or other precious material on parchments (usually tanned leather of animals) in medieval manuscripts [De Hamel, 1994, Calkins, 1983]. It is a codified representation to value its sponsor's personality and to spread explicit and implicit messages for him. This codification can be represented as a graph, which describes the illuminations' components, structured using topological position, hierarchical, semantic and metaphorical relations (e.g. the representation of animal to express a human moral value). Those latter are particularly abundant in the illuminations we are studying: the ones of the court of Burgundy's Duke.

The Duke's court was an aristocratic micro-society composed of a set of entities including the Duke himself, the earl (his eldest son), the Duchess, their wide family, the ecclesiastics (bishop, clerics), the advisors and some knights. Its territory was vast from the south of actual Burgundy to Amsterdam partially made of rural areas but also urban areas (Brussels, Bruges, Gant, Dijon, Lille, etc.). Different social groups in the network of the court (bankers, shopkeepers, academics, townspeople, etc.) operated in more or less wide networks [Clouzot et al., 2015]. The illumination in figure 1 is an example of those networks. It is composed of the Duke, his son, advisors and some knights. They constituted a closed social network symbolised by the golden fleece necklace.

In those illuminations, some objects, characters or images as a whole are considered as a sign which works as a metaphor or sometimes stronger than a metaphor. They constituted a communication channel which goes from the sponsor (the Duke for example), the author (illuminator) or the copyist of the manuscript to the target (the Duke, a politician, king/queen or other noble). The themes depicted within them express the sponsor's values, his social rank, cultural values, family or his society in the past, the present, and the future (after death). Therefore, they aim to spread messages, idealised relationships such as political power, religious values and to give an ideological representation of the sponsor although they served to illustrated scenes of events

\footnotetext{
${ }^{2}$ WWW. facebook. com

$3_{\text {WWW. }}$ Iinkedin. com

${ }^{4}$ wWw. viadeo. com

${ }^{5}$ https: / / twitter.com
} 
in the court such as banquets, weddings or balls. Those scenes and their meanings change with the design's context and the messages to covey. In this design process, they are selected by the illuminator (the person who paints the images) according to the recipient's choices and drawn as images which are published in other political events such as the luxurious manuscript's donation to the Duke (scene described in the illumination of figure 1), banquet or knight's meeting.

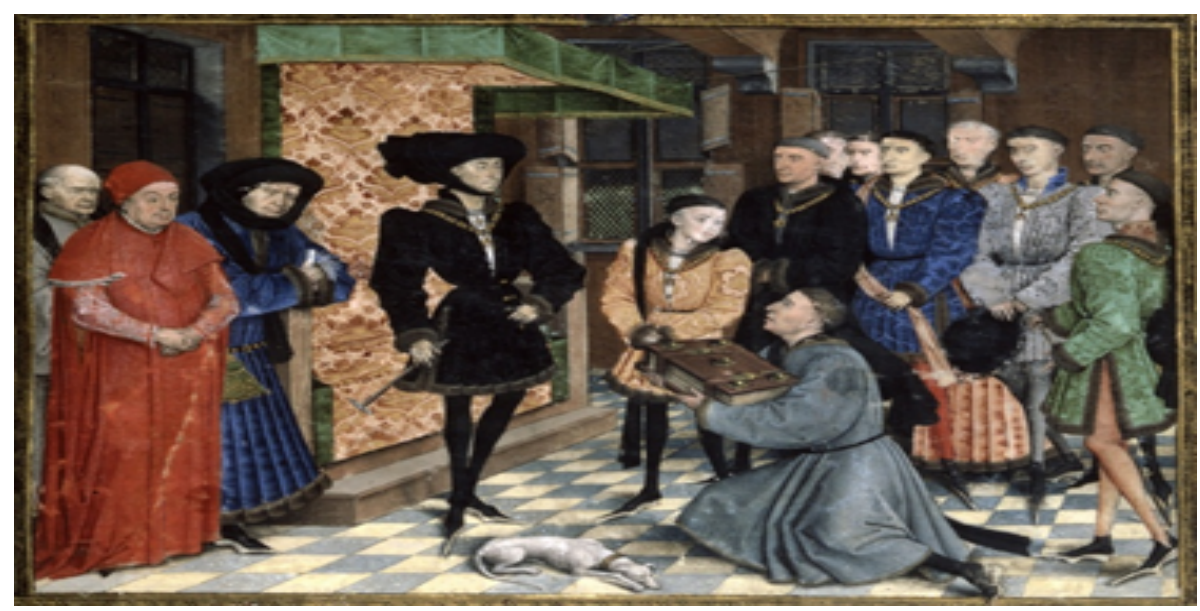

Figure 1: An illumination which illustrates the donation's scene of a luxurious illuminated manuscript to the Burgundy Duke, Philippe the Good. Brussels, Royal Library of Belgium, ms. 9243, folio 185 verso, Chronicles of Hainaut by Jean Wauquelin, 1446

\subsection{Correspondence between illuminations and social media}

Regard to all these virtues and uses, an illuminated manuscript is a medieval model of the current social media as Twitter or LinkedIn, since it is a communication and resources sharing tool.

However, an illumination itself is an animation or upkeep resource of a social network (the Duke's social network) insofar as it tells and shares a history with the social network's members. The goal is then to show the Duke's influence and to convey it. Some signs of that influence could be seen through some relations in the illumination such as:

- the allegiance of the court to the Duke: the advisors, the illuminator, the earl, the knights (except the one in green clothes), and the greyhound are faithfuls to the Duke. Their topological position (e.g. the advisors are stand up behind the Duke, the illuminator is knelt down in front of the Duke) in the image in figure 1 indicate this implicit and influential relationship;

- the wealth of the Duke through the purses he wears and his outfit;

- the power of the Duke: he holds a command stick and is stand up in the center of the image.

\section{RELATED WORKS}

The knowledge representation using ontologies is not new and several works treat it in different domains. For the social networks' modelling, a famous ontology has been created: the FOAF ${ }^{6}$ ontology. That famous ontology's authors designed it after a deep analysis of (online) social

\footnotetext{
${ }^{6}$ FOAF: Friend of A Friend is a popular ontology which describes the social relations between entities as well as their interests in a social network
} 
network platforms. They gathered and reviewed information from the activities, events, documents (images, videos, etc.) that the network's members publish on those platforms. After understanding how these users were organised and how they communicate each with others through the published information, the FOAF ontology has been built and it is actually the data set which represents the social networks platforms. Its specification is detailed in [Brickley and Miller, 2007].

FOAF has been used by many authors who sought to treat some issues, such as trust in recommendation systems [Golbeck et al., 2006], [Sherchan et al., 2013], security [Kruk, 2004], in the social network's platforms. To achieve that, those authors modified the FOAF ontology to include an initial trust scale in the relationships between entities. In our work, we use it to show the similarity between the illuminations and the social media and to extend our model in order to be more exact in our specification.

For the description of medieval images and cultural heritage's data in general, [Dörr, 2002] proposes an ontology (Cultural Images and Documents object-oriented Conceptual Reference Model, CIDOC-CRM) which models information on cultural heritage. It describes the concepts and relationships which structures the documents (images, sculptures, etc.) used in the cultural heritage's domain. According to the website ${ }^{7}$ dedicated to CIDOC-CRM, it contains 90 concepts and more than 140 properties, both organised with subsumption relation. That ontology is very common because it covers every single document used in the domain of cultural heritage and it helps a lot for the digitalisation of that domain's data. The ontology we propose here also contributes to this digitalisation but it is specific to the illuminations of Burgundy's Dukes. Many other works on historical documents' modelling use CIDOC-CRM in order to extend their ontology through mapping.

The [Doerr et al., 2006] extended the CRM by combining it with another ontology which describes the digital library. This promoted knowledge integration. The [Damova and Dannélls, 2011] proposed an infrastructure which enables the easy extension of a domain-specific data and the convenient querying of multiple data sets. The approach is based on a schema level and instance level alignment. That infrastructure combines several ontologies such as PROTON ${ }^{8}$ and CIDOC-CRM. The resulting ontology is applied to real data from the Gothenburg City Museum in order to handle queries on multiple data sets in the way of open linked data (LOD). Our ontology could be used in the same way. We linked it to other ontologies such as FOAF, Event $^{9}$. It could also be a data set on illuminations of the Burgundy's Duke and used as an underling ontology for a semantic information extraction's system.

Information extraction aims to retrieve some types of information from natural language text or images by processing them automatically [Wimalasuriya and Dou, 2010] or semi-automatically. For example, an information extraction system might retrieve information about artworks, made by an artist, from a database while ignoring other types of information. Ontology-based information extraction has recently emerged as a subfield of information extraction in which ontologies play a crucial role in the extraction process [Wimalasuriya and Dou, 2010]. The proliferation of resources sharing services on the Internet increased the need for images retrieval from web pages or other repositories. The main goal in image retrieval is the finding of images

\footnotetext{
${ }^{7}$ http://www.cidoc-crm.org/get-last-official-release

${ }^{8}$ PROTON: is an upper-level ontology which contains 542 classes and 183 properties. http://proton. semanticweb.org/

${ }^{9}$ http://motools. sourceforge. net/event/event.html. Event ontology is centered around the notion of the event, seen here as a set of activities performed in arbitrary time/space regions by cognitive agents.
} 
based on their semantic content such as their topic or contents (objects, persons, etc.). There are two major image retrieval paradigms which attempt to capture this goal: text-based metadata image retrieval and content-based image retrieval (CBIR) [Manzoor et al., 2012]. According to the goal mentioned above, CBIR is the most used and is based on ontologies for the easy description of image's contents and the simple calculation of their similarity which enables a user to find exact and related results in images extraction process according to the contents. CBIR approach is used in [Manzoor et al., 2012] to propose a system through which users will find relevant images when facing a repository of images whose content is complicated and partially unknown to them. In order to achieve that, the authors provide a semantic annotation of all images stored in the repository following an ontology built for that purpose. It is also a trend in the annotation of images within social media as applied by [Ghosh and Bandyopadhyay, 2017] for the semantic annotation of images within a social media in order to facilitate their retrieval, semantically combination, and reuse by computerised systems. In the same way, our ontology could be used as support for images (illuminations) semantic annotation in order to retrieve relevant illuminations according to their contents from a repository. To do so, semantic web technologies are used.

Considering the cultural heritage's data as syntactically and semantically very heterogeneous, multi-languages, semantically very rich and highly connected because they are produced by different entities (museums, archives, archaeological digs, etc.), [Hyvönen, 2012] gives an insight about when, why, and how to use those technologies in practice and efficiently publish those data on the Web. The author mentions most of the formalisms we use in our work. The main reasons which motivate us in this work are the sharing of the knowledge extracted from illuminations, their interoperability and possible integration in other similar knowledge, and the provision of a valid model which could be used by computerised systems. Moreover, the particularity of the ontology we built is its frequent evolution. We can progressively integrate concepts from other illuminations through the framework we are implementing.

\section{SEMANTIC FORMALISATION OF ILLUMINATIONS}

This section is dedicated to our semantic formalisation of illuminations. It contains a brief description of the concepts and used terms as well as some of our partial results.

\subsection{Ontological modelling of illumination and the technical formalisms used}

The term ontology has many definitions in the literature because it is applicable to many fields such as philosophy, information sciences, linguistics, knowledge engineering, artificial intelligence, etc. In our project, we use the definition in [Studer et al., 1998]: "an ontology is an explicit and formal specification of a shared conceptualisation". It represents a formal conceptualisation of a domain which refers to the environment we want to describe [Gruber, 1993]. In practice, it includes a hierarchical organisation of the relevant concepts in the domain, the relations between these concepts as well as rules and axioms which constrain their operations. That conceptualisation is done using four main components (called terms) which are:

- concepts, also called classes, they correspond to the relevant abstractions of the domain retained following the finals objectives and expected applications for the ontology. A concept's name should begin with a capital character;

- relations are relevant associations between concepts. They can be hierarchical (generalisation (Up-Down)/specialisation (Bottom-Up), aggregation/composition, instance of), associative, semantic (equivalent, synonym, homonym, antonym, meronym, etc.); 
- axioms are assertions accepted as true about the domain's abstractions. They constrain the operations of the concepts and allow to infer new knowledge from the described one in the domain;

- instances constitute the ontology extensional definition. They state the static or factual knowledge of the domain.

Examples of ontological terms into the illumination in figure 1 are: for the concepts (Duke, SocialStanding, Knight, Activity, Person, Animal); for the relations of generalisation (Duke is a Person, Greyhound is an Animal); for the relations of aggregation (a Project is composed of Activities); for the relaton of synonymy (Prince is synonym of Earl); for the associative relations (Illuminator offers the Book, the Book is offered to the Duke); for the instances of concept (banquet, game, hunting, falconry are instances of activity).

The ontological representation of a domain must banish all semantic ambiguity. It should then provide an uniform and reusable knowledge support to the community of users for efficient sharing and communication. In our work, this constraint is ensured by the use of a formal language: the description logics (DL) through its variant SHOIN(D). That DL's variant is widely used in ontological representation because of its expressiveness, decidability and controlled complexity. Its constructors (the set of lexical symbols and operators used in the DL) give a sufficient expressiveness for the ontological description. Those constructors are $S(A L C$ and $R+$ ), $H, O, I, N$ [Baader et al., 2005, Baader, 2011].

- S(ALC and R+): is the name of a DL's sub-variant which groups the ALC constructors (basic DL's sub-variant composed of following operations - the definition of the global concept (Top, represented by $T$ ), the concept of nothing (bottom, $\perp$ ), every concept (Duc for example), the conjunction of concepts (Duc AND Knight, Duc $\sqcap$ Knight ), the universal quantification (All children of the Duke, $\forall c h i l d . D u c$ ), the existential quantification (the Earl's father, $\exists$ father.Earl) and the negation of a concept (not Animal, $\neg$ Animal)) and constructor R+ (it allows the composition of roles (relations). Example: father $($ father $(X, Y), Z)$ to state that $X$ is the grandfather of $Z$ where $X, Y, Z$ are concepts and father, a relation);

- H: is the constructor of the hierarchy between concepts. Example: Duc is_a Person, Duc $\subseteq$ Person;

- O: is the constructor of instances. Example: games, banquet are instances of the concept Activity, Activite\{games, banquet\};

- I: is for the inverse of a role (relation). Example: the relation child is the inverse of the relation father, child. $\top \equiv \exists$ father $^{-1}$. $\top$;

- $\mathbf{N}$ : is for the restriction of number.

Example: The Knights are up to 8, KnightNumber ${ }_{<=8}$ Knight.

Moreover, those constructors can be combined by the operators of hierarchy (subsumption, expressed by $\subseteq$ ) or equivalence $(\equiv)$ to define other concepts or organise them through the rules (or axioms).

Figure 2 shows a view of our ontology's concepts. It is modeled in Protege ${ }^{10}$. Its specification is detailed in http://medievenl.ontology. checksem. fr/.

\footnotetext{
${ }^{10}$ Protege is an ontology editor. http://protege.stanford.edu/
} 


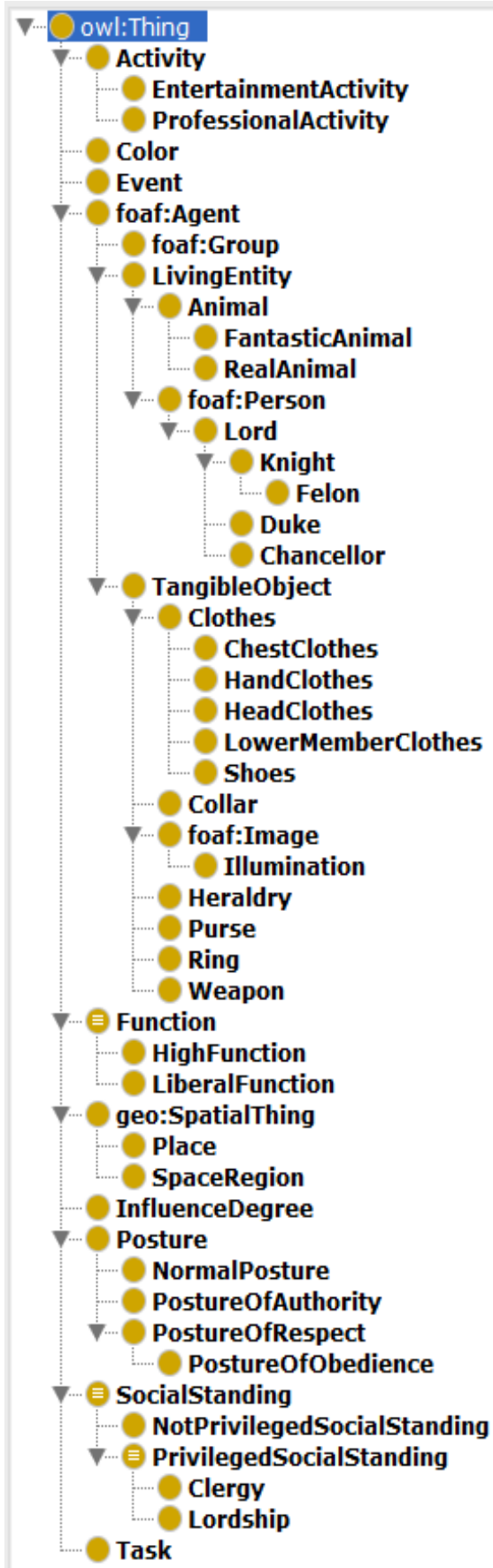

Figure 2: A view of the concepts in our ontology on the illumination in figure 1. Those concepts are organised through the subsumption relation $\left(i s_{-} a\right)$.

Once this modelling is finished, it is saved in a formal syntax's form in a language ${ }^{11}$, such as $\mathrm{RDF} / \mathrm{XML}$, turtle or OWL, generated by Protege. The final model is extensible and can be combined with others such as FOAF with which it has many common terms (e.g. foaf:Person, foaf:member, foaf:interest, foaf:Group, foaf:depict, foaf:Image).

In addition, we are developing a framework ${ }^{12}$ which enables to upload an illumination, delim-

\footnotetext{
${ }^{11} \mathrm{RDF} / \mathrm{XML}$, turtle and OWL are computer languages used in ontologies development.

${ }^{12}$ Illumination3.0, our platform (the implementation is in progress) for the annotation of medieval illuminations, http://illumination2.checksem.fr/illumination/app/accueil.
} 
ited its concepts and connect them each to others with relations. This annotation of the concepts and their relations are manually done, but it is done following a list of concepts and relations provided by the medievalists (experts in medieval illuminations analysis) for the consistency of the annotated concepts and relations. Figure 3 shows an annotated illumination within that framework. The concepts and relations annotated are saved in $\mathrm{JSON}^{13}$ file which is created to enable the extension of the ontology on its Tbox (set of concepts in the ontology and their relations) and Abox (set of instances, facts or individuals in the model).

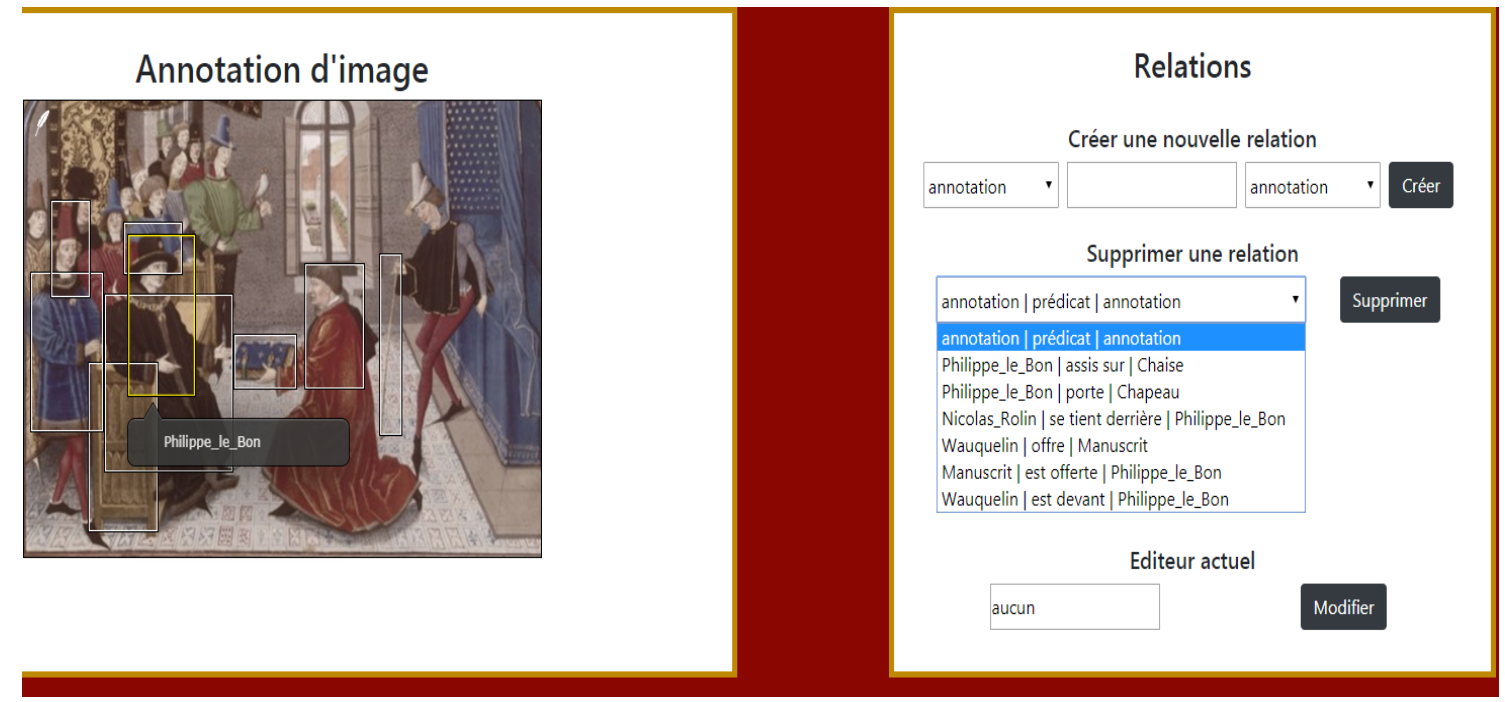

Figure 3: An interface of our framework Illumination3.0 (http: / / illumination2. checksem. fr/illumination/app/annotation) with an illumination annotated within it

The built model can be queried by SPARQL ${ }^{14}$ in order to extract targeted components and to answer specific questions such as activities represented in the illumination; who are the faithful persons to the Duke? Another interest of an ontology is its ability to discover new knowledge from the ones initially described. This discovery is ensured thanks to some logical formalisation called inference's rules and their execution within an inference engine (a tool used to make logical deductions about knowledge assets). In our work, those these rules are built in SWRL ${ }^{15}$ (Semantic Web Rules Language) in order to reason on our ontology's components. In the illumination in figure 1, some examples of symbolic relations and their formalisation with Horn clauses are in table 1.

\subsection{Example of formalisation of symbolic relations: metaphors}

The formulation of logical rules in the form of Horn's clause enables us to interpret metaphors in illuminations. A metaphor is a rhetorical figure which makes an implicit and perceptible comparison between two different concepts. Although the two concepts related by the comparison belong to different semantic fields, they share common characteristics which create an analogy between them [Perrenoud, 2002]. For example, one can praise the bravery of a man by

\footnotetext{
${ }^{13}$ JSON (JavaScript Object Notation) is a lightweight data exchange format. It is easy to read, write and analyse

${ }^{14}$ SPARQL is the language used to query an RDF (Resource Description Framework) database. It is similar to the SQL language used to query relational databases

${ }^{15}$ SWRL (Semantic Web Rules Language) is the language which allows us to build Horn clauses (logical rules of inferences). It helps to extend the expressiveness of some OWL's (Ontology Web Language) variants. It allows them to create complex rules. OWL is another language dedicated to the ontology's development. It has many variants: OWL-DL (corresponds to SHOIN (D)), OWL-Lite (uses fewer constructors), OWL-Full (Complete OWL, which is undecidable).
} 


\begin{tabular}{|c|c|}
\hline Symbolic relationships & Formalisation in Horn Clauses \\
\hline A knight has the right to kill & hasRightToKill(X):- Knight(X) \\
\hline $\begin{array}{l}\text { A knight is a monk wearing } \\
\text { the necklace of the Golden } \\
\text { Fleece }\end{array}$ & $\operatorname{Knight}(X):-\operatorname{Monk}(X)$, GoldenNecklace $(Y)$, wear $(X, Y)$ \\
\hline $\begin{array}{l}\text { A person carrying a weapon } \\
\text { has the right to kill }\end{array}$ & hasRightToKill $(X):-\operatorname{Person}(X), \operatorname{Weapon}(Y), \operatorname{carry}(X, Y)$ \\
\hline $\begin{array}{l}\text { We are obedient to a person } \\
\text { in front of whom we stand in } \\
\text { a obedient position }\end{array}$ & $\begin{array}{l}\text { beingObedient }(X, Y) \text { :- Being }(X), \text { Person }(Y) \text {, ObedientPosi- } \\
\text { tion }(Z), \text { beIn }(X, Z)\end{array}$ \\
\hline $\begin{array}{l}\text { Being obedient to someone } \\
\text { implies to be faithful to him }\end{array}$ & faithful $(X, Y)$ :- beingObedient $(X, Y)$ \\
\hline A felon wears green clothes & $\begin{array}{l}\text { beingFelon }(X):-\operatorname{Person}(X), \quad \text { Clothes }(Y), \text { GreenColor }(Z) \text {, } \\
\text { hasColor }(Y, Z) \text {, wear }(X, Y)\end{array}$ \\
\hline $\begin{array}{l}\text { A person in mourning dresses } \\
\text { all in black }\end{array}$ & $\begin{array}{l}\text { beingInMourning }(X):-\quad \text { person }(X), \quad \text { Clothes }(Y), \quad \text { Black- } \\
\text { Color }(Z), \text { hasColor }(Y, Z), \text { wear }(X, Y)\end{array}$ \\
\hline
\end{tabular}

Table 1: Some examples of symbolic and implicit relations in the illumination depicted in figure 1 and their formalisation with Horn Clauses. We suppose that all the explicit concepts and relations in the right side of a clause are clearly defined. Examples: GreenColor $(X)$ represents the green color, hasColor $(X, Y)$ states a thing $\mathrm{X}$ has the color $\mathrm{Y}, \operatorname{Monk}(X)$ is the concept which represents a monk, etc.

designating him by a lion, but man and lion remain concepts which are totally different (man is a human while the lion is an animal). Although there is no real consensus from linguists on an universal typology of metaphors, two main types can be enumerated: metaphor in praesentia and metaphor in absentia [Perrenoud, 2002].

In a metaphor in praesentia [Perrenoud, 2002], the two concepts (compared and comparing) are presents and it is possible to perceive quite easily their link. That makes the comparison less allusive and relatively attenuates the power of the metaphor. Example: "The butterfly, flower without stem" (Nerval). The butterfly is compared to a flower to enhance its splendor. This metaphor can be expressed in description logics by:

$$
\begin{aligned}
\text { Flower } & \equiv \text { Butterfly } \\
\text { Flower } \sqcap \text { Butterfly } & \equiv \exists \text { beingSplendid. } \top
\end{aligned}
$$

The metaphor in absentia [Perrenoud, 2002] is characterised by the unique presence of the comparing concept and the absence of the compared one whose existence is insinuated by the context. Example: "My bitter mind, a worried and crazy wing flies over the sea" (Verlaine). The comparing "mind" is clearly expressed while the compared "bird" can be guessed through the words "wing" and "fly" which are part of its lexical field. It is a metaphor which is not openly expressed. The link between the comparing and compared concept is made by logical inference. This example can be expressed in description logics as shown below:

$$
\begin{gathered}
\text { Bird } \subseteq \exists \text { hasWing. } \top \sqcap \exists \text { canFly. } \top \\
\exists \text { hasWing. } \top \sqcap \exists \text { canFly. } \top \equiv \text { Mind } \sqcap \text { Lightness. }
\end{gathered}
$$

By transitivity we could deduce that Bird $\subseteq$ Mind $\sqcap$ Lightness. 
Besides the textual form, the expression of a metaphor can be visual. For example, there are images which contain metaphors observable through shapes or symbols that embody phenomena, events, characters, or other. As incoming work, we will formalise the expression of visual metaphors within our medieval illuminations by the means of SWRL rules.

\section{CONCLUSION AND FUTURE WORKS}

This paper presented the application of knowledge engineering's techniques to medieval illuminations of Burgundy's Duke. First, we identified and showed the process of design and distribution of those illuminations as a medieval model of a social network which obeyed the same motivations and codes as the current social media. Nevertheless, this system of expression uses more complex types of relations such as metaphors. Second, we proposed a formal ontology for those illuminations. That ontology described in SHOIN(D) allows the medievalists and the general public to annotate the components of illuminations using a Web framework and to encode the reasoning associated to metaphors in the form of logical rules.

As future work, we plan to combine our ontology with a system which semantically represents the current social media. The goal is to eventually propose a functional extension of those social media and improve the calculation of the influence of their users through qualitative analysis.

\section{Acknowledgments}

This work is funded by the CNRS as part of a PEPS project, the French Embassy in Bamako and the Malian government for the co-funding of a thesis, the Burgundy Franche-Comté, and the University of Burgundy. We would like to thank all these institutions as well as Mr. Rafik Zebidi and Mr. Florian Lacroix for their help in the metaphors formalisation and implementation parts.

\section{References}

Franz Baader. What?s new in description logics. Informatik-Spektrum, 34(5):434-442, 2011.

Franz Baader, Ian Horrocks, and Ulrike Sattler. Description logics as ontology languages for the semantic web. In Mechanizing Mathematical Reasoning, pages 228-248. Springer, 2005.

Eytan Bakshy, Itamar Rosenn, Cameron Marlow, and Lada Adamic. The role of social networks in information diffusion. In Proceedings of the 21st international conference on World Wide Web, pages 519-528. ACM, 2012.

Dan Brickley and Libby Miller. Foaf vocabulary specification 0.91, 2007.

Robert G Calkins. Illuminated books of the middle ages. Thames and Hudson London, 1983.

Martine Clouzot, Rafik Zebidi, and Christophe Nicolle. (poster session): Characterization of semantic relations in medieval illuminations. In Proceedings of the 1rst International Conference of Science and Engineering of Arts Heritage and Archaeology, number 1. SEAHA, 2015.

Mariana Damova and Dana Dannélls. Reason-able view of linked data for cultural heritage. In Third International Conference on Software, Services and Semantic Technologies S3T 2011, pages 17-24. Springer, 2011.

Christopher De Hamel. A history of illuminated manuscripts. Phaidon Press London, 1994.

Martin Doerr, Jane Hunter, and Carl Lagoze. Towards a core ontology for information integration. Journal of Digital information, 4(1), 2006.

Martin Dörr. The cidoc crm-an ontological approach to semantic interoperability of metadata, 2001. AI Magazine, Special Issue on Ontologies, Nov, 2002.

Mrs Sayantani Ghosh and Samir K Bandyopadhyay. A proposed method for semantic annotation on social media images. International Journal Of Engineering And Computer Science, 6(6), 2017.

Jennifer Golbeck, James Hendler, et al. Filmtrust: Movie recommendations using trust in web-based social networks. In Proceedings of the IEEE Consumer communications and networking conference, volume 96, pages 282-286, 2006.

Tom Gruber. What is an ontology. WWW Site http://www-ksl. stanford. edu/kst/whatis-an-ontology. html (accessed on 07-09-2004), 1993.

Eero Hyvönen. Publishing and using cultural heritage linked data on the semantic web. Synthesis Lectures on the Semantic Web: Theory and Technology, 2(1):1-159, 2012. 
Sebastian R Kruk. Foaf-realm-control your friendsâĂŹ access to the resource. In FOAF Workshop proceedings, volume 186, 2004.

Damien Leprovost, Lylia Abrouk, and David Gross-Amblard. Discovering implicit communities in web forums through ontologies. Web Intelligence and Agent Systems: An International Journal, 10(1):93-103, 2012.

Umar Manzoor, Naveed Ejaz, Nadeem Akhtar, Muhammad Umar, M Shoaib Khan, and Hafsa Umar. Ontology based image retrieval. In Internet Technology And Secured Transactions, 2012 International Conference for, pages 288-293. IEEE, 2012.

Eugenio Martínez-Cámara, M Teresa Martín-Valdivia, L Alfonso Urena-López, and A Rturo Montejo-Ráez. Sentiment analysis in twitter. Natural Language Engineering, 20(1):1-28, 2014.

Philippe Perrenoud. D'une métaphore à l'autre: transférer ou mobiliser ses connaissances? In L'énigme de la compétence en éducation, pages 45-60. De Boeck Supérieur, 2002.

Elie Raad. Découverte des relations dans les réseaux sociaux. $\mathrm{PhD}$ thesis, Dijon, 2011.

Faneva Ramiandrisoa and Josiane Mothe. Profil utilisateur dans les réseaux sociaux: Etat de l'art. In CORIA, pages 395-404, 2017.

Wanita Sherchan, Surya Nepal, and Cecile Paris. A survey of trust in social networks. ACM Computing Surveys (CSUR), 45(4):47, 2013.

Rudi Studer, V Richard Benjamins, and Dieter Fensel. Knowledge engineering: principles and methods. Data \& knowledge engineering, 25(1-2):161-197, 1998.

Stanley Wasserman and Katherine Faust. Social network analysis: Methods and applications, volume 8. Cambridge university press, 1994.

Daya C Wimalasuriya and Dejing Dou. Ontology-based information extraction: An introduction and a survey of current approaches, 2010. 